\title{
Investigation of Di-ethylhexyl Phthalate Migration by Applying Magnetic Solid Phase Extraction Method Followed by GC-FID Determination
}

\author{
Mohammad Faraj ${ }^{1, *}$, Mahnaz Adeli $^{2}$ \\ 1- Faculty of Food Industry and Agriculture, Department of Food Science \& Technology, Standard Research Institute (SRI), Karaj, Iran. \\ 2- Knowledge Development \& University Relationship Department, Iran Khodro Company, Tehran, Iran.
}

Received: July 2016

Accepted: September 2016

\section{A B S T R A C T}

Background and Objectives: The addition of plasticizers (mainly phthalates and adipates) makes plastic flexible and popular for packaging, storing and preserving food, water, and so on. The most widely used plasticizer in food contact applications is di-ethylhexyl phthalate (DEHP) though being suspected to have carcinogenic and estrogenic properties. The aim of this study is application of magnetic solid phase extraction as new absorbent for determination of DEHP in water samples by gas chromatography (GC).

Materials and Methods: Important factors in extraction, separation and determination processes were optimized using the one-variable-at-a-time method. For optimization, all tests were performed two times. Figures of merit of the proposed method were evaluated. The amount of DEHP in some water samples was determined using the proposed method.

Results: The results showed that the obtained chromatogram of extract was free of significant interference. The preservatives' recoveries ranged from $91.6 \%$ to $102.2 \%$. Limit of detection and limit of quantitation were 0.1 $\mathrm{mg} \mathrm{kg}^{-1}$ and $0.3 \mu \mathrm{g} \mathrm{L}^{-1}$, respectively. Concentration of DEHP in the studied samples was in the range of N.D$2.3 \mu \mathrm{g} \mathrm{L}{ }^{-1}$.

Conclusions: The acceptable performance and reliability of the proposed method were demonstrated. Temperature and time were fount as the most effective parameters in migration of DEHP.

Keywords: Di-ethylhexyl phthalate, Magnetic solid phase extraction, Migration, Gas chromatography

\section{Introduction}

Phthalate esters (PEs) are used primarily as plasticizers in polymeric materials to increase their flexibility through secondary weak molecular interactions with polymer chains. Since they are physically bound to the polymer chains, PEs may be released into the environment during the production, use, or incineration of polymeric materials containing them $(1,2)$. Due to the potential risks of PEs to human health and the environment $(3,4)$, several of them have been included in the list of priority pollutants by different national and international organizations. For example, the United States (US) Environmental Protection Agency (EPA) has established a maximum admissible concentration (MAC) of $6 \mu \mathrm{gL}^{-1}$ for di-ethylhexyl phthalate
(DEHP) in water (5). DEHP is the most widely used $\mathrm{PE}$ in the world, which represents a quarter of total production of plasticizers (6).

To determine the plasticizers in water samples, gas chromatography (GC) $(8,9)$, gas chromatographymass spectrometry (GC-MS) $(7,10,11)$ and highperformance liquid chromatography (HPLC) (12-14) have been used. Nevertheless, when the concentration levels are low, a previous enrichment step is usually needed. The pre-concentration techniques, which are commonly applied to monitor phthalates in water, are solid-phase microextraction (SPME) (9), hollow-fiber liquid-phase microextraction (HF-LPME) (9), homogeneous liquid-liquid extraction (HLLE) (7), solidification of floating drop microextraction 
(SFDME) (7), single drop microextraction (SDME) (8), vortex-assisted surfactant-enhancedemulsification liquid-liquid microextraction (10), dispersive liquid-liquid microextraction (DLLME) (11-14), and solid-phase extraction (SPE) (15).

More recently, the use of magnetic NPs in solid phase extraction (named magnetic solid phase extraction (M-SPE)) is gaining many researchers' interest (16-18). M-SPE involves the addition of magnetic sorbent particles to the sample solution. The target compound is adsorbed onto the magnetic material, and the magnetic particle (containing the analyte) is then separated from the sample solution by the application of an external magnetic field. Finally, the analyte is recovered from the adsorbent by elution with the appropriate solvent, which is subsequently analyzed. This approach has several advantages over traditional solid phase extraction: (i) it avoids timeconsuming and tedious on-column SPE procedures, (ii) it provides a rapid and simple analyte separation that avoids the need for centrifugation or filtration steps, (iii) the magnetic sorbents synthesized to date have high selectivity, even when complex environmental or biological matrices are exist, and (iv) since the majority of sample impurities are diamagnetic, they do not interfere with magnetic particles during the magnetic separation.

The aim of the present study was to develop an NPbased method for the pre-concentration and determination of trace amounts of DEHP. The method is based on the extraction of DEHP on octadecylfunctionalized magnetic silica nanoparticles (C18-f$\mathrm{m}$-Si-NPs), as SPE sorbent. The level of DEHP in the extracting phase was determined by gas chromatography-flame ionization detection (GCFID). To the best of our knowledge, this method has not been employed previously in the extraction and determination of trace amounts of DEHP from aqueous samples.

\section{Materials and Methods}

Reagents and standards: The analytical standards of DEHP, pyrene (as an internal standard), acetone, acetonitrile, and methanol (HPLC grade) as well as $\mathrm{NaCl}$ were acquired from Merck Company (Darmstadt, Germany). Pyrene was utilized as an internal standard. The stock solution of DEHP was prepared in methanol at the concentration of $100 \mathrm{mg}$ $\mathrm{L}^{-1}$. The stock solutions were kept in the refrigerator $\left(2-8{ }^{\circ} \mathrm{C}\right)$ at least for three months. Additional standard solutions were daily made from the appropriate dilutions of these stock solutions with deionized distilled water. Octadecyl-functionalized magnetic silica nanoparticles were prepared from the Yamini's research group as they synthesized and well characterized the nanoparticles previously (19).

Gas chromatography flame ionization detection analysis: Chromatographic analyses were done on an Agilent 7890 gas chromatograph (GC) (Centerville Road, Wilmington, USA) equipped with a flame ionization detector (FID). Separation of the analytes was performed on a HP-5 fused-silica capillary column $(30 \mathrm{~m} \times 0.32 \mathrm{~mm}$, i.d.; $0.25 \mu \mathrm{m}$, film thickness) from Supelco (Bellefonte, PA, USA). The temperature of the detector was $280^{\circ} \mathrm{C}$; the detector was fed with hydrogen, air, and nitrogen (as auxiliary gas) at the flow rates of $45 \mathrm{~mL} \mathrm{~min}{ }^{-1}, 300 \mathrm{~mL} \mathrm{~min}^{-1}$ and $30 \mathrm{~mL} \mathrm{~min}^{-1}$, respectively, which were generated by multiple CLAIND gas generators (Lenno, Italy). The GC split valve was opened with the split ratio of $1: 5$, and helium (99.999\% purity) was exploited as carrier gas at a constant flow rate of $4 \mathrm{~mL} \mathrm{~min}^{-1}$. The column temperature program was as follows: the oven temperature was held at $120^{\circ} \mathrm{C}$ for $2 \mathrm{~min}$, then raised to $280^{\circ} \mathrm{C}$ at a rate of $25^{\circ} \mathrm{C} \mathrm{min}{ }^{-1}$, and finally, held at $280^{\circ} \mathrm{C}$ for $5 \mathrm{~min}$. The analytical signal was taken as the relative peak areas of the plasticizers to the peak area of the internal standard (pyrene).

Magnetic solid phase extraction procedure: Firstly, $45 \mathrm{mg}$ of the C18-f-m-Si-NPs (dispersed in $1.0 \mathrm{~mL}$ methanol) was added to $200 \mathrm{~mL}$ of the $100 \mu \mathrm{gL}^{-1}$ spiked water sample (containing pyrene as internal standard with concentration of $500 \mu \mathrm{g} \mathrm{L}^{-1}$ ) and homogeneously dispersed with the aid of ultrasonication for $2 \mathrm{~min}$. Subsequently, a strong $\mathrm{Nd}-$ Fe-B magnet $(5 \mathrm{~cm} \times 3 \mathrm{~cm} \times 2 \mathrm{~cm}, 1.4 \mathrm{~T})$ was deposited at the bottom of the beaker. After about 5 min, the suspension became limpid; then it was decanted. The preconcentrated target analyte was eluted from the isolated sorbent with $1.0 \mathrm{~mL}$ of methanol, and $2 \mu \mathrm{L}$ of the solution was injected into the GC-FID system. A univariate approach was employed to optimize the influential factors in this method.

\section{Results}

\section{Optimization of magnetic solid phase extraction}

Effect of the amount of adsorbent: In order to explore the impact of adsorbent, 10-60 $\mathrm{mg}$ of the 
C18-f-m-Si-NPs was added to $200 \mathrm{~mL}$ of the sample solution. The acquired results showed that by increasing the sorbent amount from $10 \mathrm{mg}$ to $45 \mathrm{mg}$, the ER\% slowly increased and then remained constant owing to a rise in the number of accessible sites (Fig. 1). Consequently, $45 \mathrm{mg}$ of the C18-f-m-Si-NPs was utilized in the next experiments.

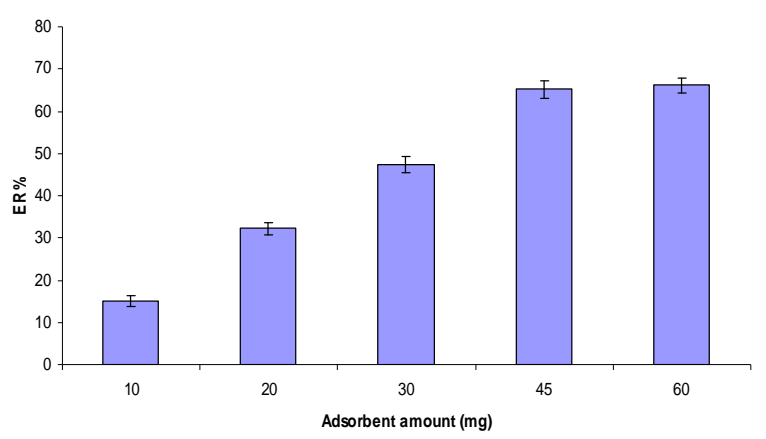

Fig. 1. Effect of adsorbent amount on the extraction efficiency of DEHP

Effect of ionic strength: To examine the influence of ionic strength on the quantitative m-NP-based SPE of the plasticizer, the experiments were done by adding $\mathrm{NaCl}$ (in the concentration range of $0-10 \%$ w/v) to the sample solution. The results demonstrated that when $\mathrm{NaCl}$ concentration was increased up to $5 \%$ w/v, the ERs\% of DEHP improved because of the salting-out effect (Fig. 2). At higher concentrations of $\mathrm{NaCl}$, the signals diminished; hence, in the subsequent experiments, $5 \%(\mathrm{w} / \mathrm{v}) \mathrm{NaCl}$ was added to the sample solution.

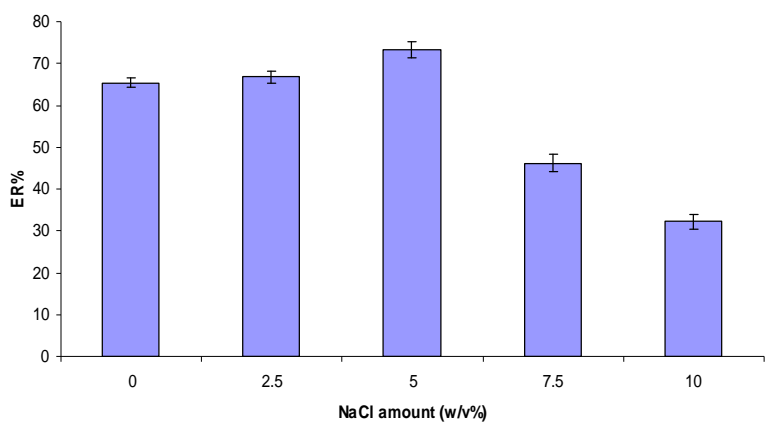

Fig. 2. Effect of $\mathrm{NaCl}$ amount on the extraction efficiency of DEHP

Effect of extraction time: To study the effect of extraction time on the m-NP-based SPE step, this parameter was investigated in the range of 0.5-5 min. The results established that by lengthening the extraction time up to $2 \mathrm{~min}$, the ERs\% of DEHP slightly improved and then stayed constant. Under these circumstances, the most time-consuming stage was the magnetically assisted separation of the sorbent, which took almost $5 \mathrm{~min}$. Therefore, $2 \mathrm{~min}$ was chosen as the extraction time for the next experiments.

Evaluation of desorption conditions: In order to elution of DEHP from surface of the adsorbent, different solvents including acetone, acetonitrile and methanol were selected. The adsorbent was eluted using $1.0 \mathrm{~mL}$ of each eluting solvent. The results, depicted in Fig. 3, revealed that the recoveries using acetone, acetonitrile, and methanol (as eluting solvents) are 45, 62 and 75, respectively, implying that methanol is better than acetonitrile and acetone. Consequently, methanol was chosen as the eluting solvent. To evaluate the required volume of methanol to elute all the DEHP from the adsorbent surface, elution was carried out three times with $1.0 \mathrm{~mL}$ of methanol. It was concluded that a volume of $1.0 \mathrm{~mL}$ was sufficient to desorb the trapped plasticizer from the sorbent surface. Further studies also indicated that most of the adsorbed analytes ( 90\%) could be straight forwardly desorbed by a one-step elution procedure with methanol. Besides, no carryover was observed after three rounds of elution. Thus, the sorbent could be reused. It was revealed that the adsorbent could be employed in at least three successive extractions without a substantial change in its efficiency.

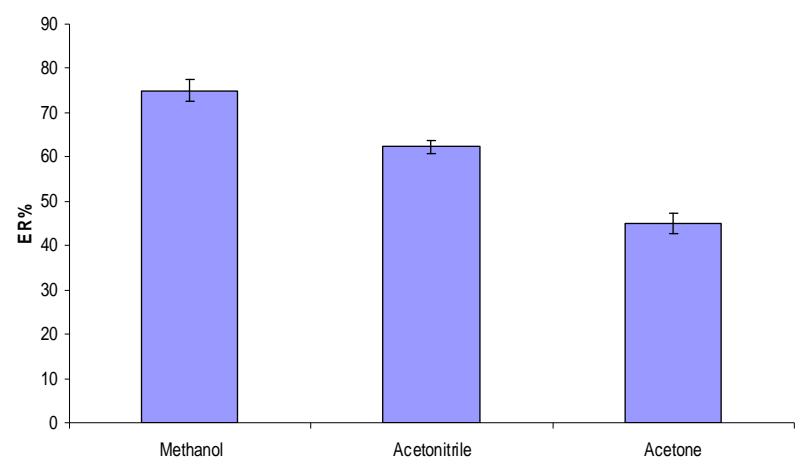

Fig. 3. Effect of type of eluent on the extraction efficiency of DEHP

In order to figure out the optimal desorption time in the m-NP-based SPE stage, desorption time was scrutinized in the range of 1-5 min at 1 min intervals. To enhance the recoveries, the adsorbent was sonicated throughout each desorption process. The 
obtained results verified that the desorption time had no significant impact on the desorption efficiency.

Sample volume: In order to study the sample volume, extraction of plasticizer was explored with different volumes of water samples ranging from 200 to $600 \mathrm{~mL}$ at $100-\mathrm{mL}$ intervals. It was found that the best extraction recoveries were almost constant when the sample volumes were $\leq 400 \mathrm{~mL}$; however, for the volumes $>400 \mathrm{~mL}$, the extraction recovery slightly diminished. For this reason, a sample volume of 400 $\mathrm{mL}$ was selected as the ideal volume for trace analysis in water samples.

Method performance: The figures of merit in the proposed method, including linear dynamic range (LDR), limit of detection (LOD), and limit of quantification (LOQ), intra and inter-day precisions, enrichment factor (EF) and extraction recovery (ER) for the extraction of DEHP from water samples were investigated under optimum conditions $(\mathrm{V}=400 \mathrm{~mL})$. The obtained results are shown in Table 1. Calibration curve was plotted using 10 spiking levels of DEHP in concentrations ranging from 0.5 to $500 \mu \mathrm{g} \mathrm{L}^{-1}$, and the good determination coefficient $\left(\mathrm{R}^{2}\right)$ of 0.9992 was obtained. For each level, three replicate extractions were performed under optimum conditions. LOD values were calculated based on the signal-to-noise ratio of $3(\mathrm{LOD}=3 \times \mathrm{S} / \mathrm{N})$; LOD was $0.1 \mu \mathrm{g} \mathrm{L}^{-1}$ and LOQ based on the signal-to-noise ratio of 10 (LOQ = $10 \times \mathrm{S} / \mathrm{N})$ was obtained $0.3 \mu \mathrm{g} \mathrm{L}^{-1}$. The intra-day precision of the proposed method was obtained 3.2 and 2.6 at 10 and $100 \mu \mathrm{g} \mathrm{L}^{-1}$ levels of DEHP, and inter-day precision of the proposed method was obtained $6.7 \%$ and $5.4 \%$ at 10 and $100 \mu \mathrm{g} \mathrm{L}^{-1}$ levels of DEHP, respectively.
The enrichment factor (EF) and extraction recovery (ER\%) were exploited to evaluate the efficiency of the suggested m-NP-based SPE. The EF was defined as the ratio of the concentration of analyte in the sediment phase $\left(C_{\text {sed }}\right)$ to the initial concentration of analyte $\left(C_{0}\right)$ in the sample:

$$
E F=\frac{C_{\text {sed }}}{C_{0}} .
$$

The ER\% was defined as the percentage of total analyte moles $\left(\mathrm{n}_{0}\right)$ extracted to the sediment phase $\left(\mathrm{n}_{\text {sed }}\right)$ :

$E R \%=\frac{n_{\text {sed }}}{n_{0}} \times 100=\frac{C_{\text {sed }} \times V_{\text {sed }}}{C_{0} \times V_{a q}} \times 100$

Where, $V_{\text {sed }}$ and $V_{\text {aq }}$ are the volumes of sediment phase and sample solution, respectively.

The EF of the DEHP was 350. Meanwhile, the ERs\% of DEHP was $78 \%$. Based on the obtained results, it can be concluded that the present technique has a high sensitivity, a broad linear range, and favourable precision.

Real sample analysis: Various water samples, comprising freshly bottled mineral water (Polour, Iran), bottled mineral water after the expiration date, water exposed to polyethylene glass and boiled water already cooled down inside a polyethylene glass, were chosen as real samples to evaluate the applicability of the suggested technique under the optimum conditions. No filtration or any extra treatment was done for any of the samples before extraction.

The relative recoveries (\%) and the RSDs\% for the analysis of DEHP in real water samples based on three-replicate extractions and determinations using the method are tabulated in Table 2.

Table 1: Figures of merit of the method for extraction and determination of DEHP

\begin{tabular}{|c|c|c|c|c|c|c|c|c|c|}
\hline \multicolumn{4}{|c|}{ RSD \% } & \multirow{3}{*}{$\begin{array}{c}\mathrm{LOQ} \\
\left(\mu \mathrm{g} \mathrm{L}^{-1}\right)\end{array}$} & \multirow{3}{*}{$\begin{array}{c}\text { LOD } \\
\left(\mu \mathrm{g} \mathrm{L}^{-1}\right)\end{array}$} & \multirow[t]{3}{*}{$\mathrm{EF}$} & \multirow[t]{3}{*}{ ER\% } & \multirow{3}{*}{$\begin{array}{c}\text { LDR } \\
\left(\mu \mathrm{g} \mathrm{L}^{-1}\right)\end{array}$} & \multirow[t]{3}{*}{$\overline{\mathrm{R}^{2}}$} \\
\hline Intra-d & $=6)$ & Inter- & $(n=6)$ & & & & & & \\
\hline $\begin{array}{c}10 \\
\left(\mu \mathrm{g} \mathrm{L}^{-1}\right)\end{array}$ & $\begin{array}{c}100 \\
\left(\mu \mathrm{g} \mathrm{L}^{-1}\right)\end{array}$ & $\begin{array}{c}10 \\
\left(\mu \mathrm{g} \mathrm{L}^{-1}\right)\end{array}$ & $\begin{array}{c}100 \\
\left(\mu \mathrm{g} \mathrm{L}^{-1}\right)\end{array}$ & & & & & & \\
\hline $3.2 \%$ & $2.6 \%$ & $6.7 \%$ & $5.4 \%$ & 0.3 & 0.1 & 350 & 78 & $0.5-500$ & 0.9952 \\
\hline
\end{tabular}

EF: Enrichment Factor; LOD: Limit Of Detection; RSD\%: Relative Standard Deviation; LDR: Linear Dynamic Range; $\mathrm{R}^{2}$ : Correlation Coefficient; ER\%: Extraction Recovery. 
Table 2. Determination of plasticizers in different water samples using the method

\begin{tabular}{lc}
\hline Sample & Concentration \pm SD $\left(\mu \mathrm{L} \mathrm{L}^{-1}, \mathrm{n}=3\right)$ \\
\hline Bottled mineral water & DEHP \\
Non-spiked & N.D \\
Spiked $\left(1.0 \mu \mathrm{g} \mathrm{L}^{-1}\right)$ & $0.93 \pm 0.05$ \\
Recovery $(\%)$ & 93 \\
Bottled mineral water after expiration date & \\
Non-spiked & $1.2 \pm 0.05$ \\
Spiked $\left(1.0 \mu \mathrm{g} \mathrm{L}{ }^{-1}\right)$ & $2.25 \pm 0.13$ \\
Recovery $(\%)$ & 102.2 \\
Water in polyethylene glass & \\
Non-spiked & $0.2 \pm 0.01$ \\
Spiked $\left(1.0 \mu \mathrm{g} \mathrm{L}^{-1}\right)$ & $1.1 \pm 0.06$ \\
Recovery $(\%)$ & 91.6 \\
Boiled water cooled in polyethylene glass & \\
Non-spiked & $2.3 \pm 0.07$ \\
Spiked $\left(1.0 \mu \mathrm{g} \mathrm{L}{ }^{-1}\right)$ & $3.1 \pm 0.22$ \\
Recovery $(\%)$ & 93.9 \\
\hline DEHP: Di-ethylhexyl phthalate &
\end{tabular}

The results demonstrated desirable accuracy for all the analyzed water samples. The RSDs\% for the extraction and determination of DEHP in the explored real water samples lay in the range of 3.1-7.3\%. Also the relative recoveries for the spiked real samples were in the range of $91.6-102.2 \%$. It is worth noting that three of the plasticizers were detected at $\mu \mathrm{g} \mathrm{L}^{-1}$ levels in the water samples, which implies the sensitivity of the suggested technique. Figure 4 depicts the m-NP-based SPE-GC-FID chromatograms of DEHP in the boiled water, already cooled down inside a polyethylene glass, before (4a) and after (4b) spiking the sample with the plasticizers at $1.0 \mu \mathrm{g} \mathrm{L}^{-1}$ level.
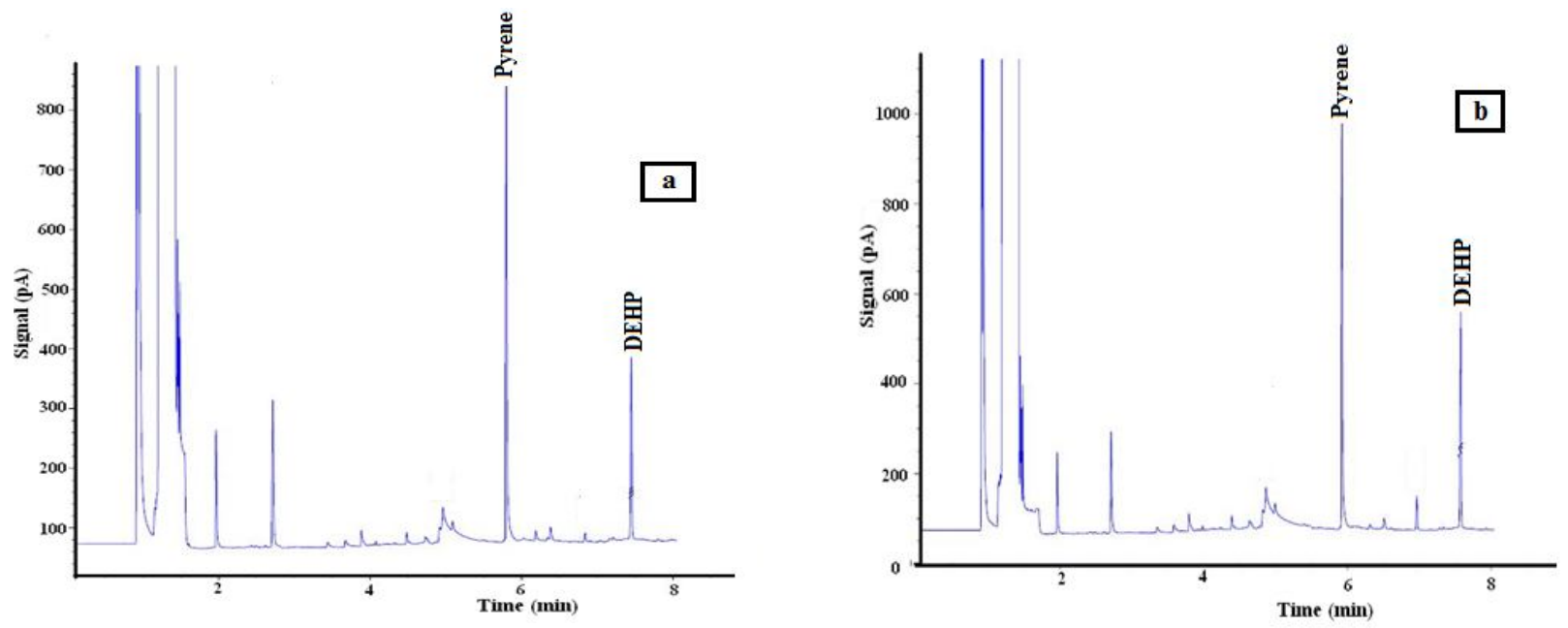

Fig. 4. GC-FID chromatograms of boiled water already cooled down inside a polyethylene glass, before (a) and after (b) spiking with $1.0 \mu \mathrm{g} \mathrm{L}^{-1}$ of the plasticizer. 
Mohammad Faraj \& Mahnaz Adeli: Education and knowlege in fasting type 2 diabetic patients

Table 3. Comparison of our proposed method with some of the techniques reported in the literature for extraction and determination of plasticizers

\begin{tabular}{|c|c|c|c|c|c|c|}
\hline Method & $\begin{array}{c}\text { LOD } \\
\left(\mathrm{ng} \mathrm{L}^{-1}\right)\end{array}$ & $\begin{array}{c}\text { DLR } \\
\left(\mu \mathrm{g} \mathrm{L}^{-1}\right)\end{array}$ & $\mathrm{EF}$ & $\begin{array}{l}\text { Sample volume } \\
\text { (mL) }\end{array}$ & RSD \% & Ref. \\
\hline SPME-GC-MS & $3-10$ & $0.1-10$ & - & 5.0 & $4-11$ & (9) \\
\hline HLLE-GC-FID & $10-20$ & $0.2-5.0$ & $472-551$ & 5.0 & $4.5-6.9$ & (7) \\
\hline SFDME-GC-FID & $10-30$ & $0.2-10$ & $162-198$ & 20.0 & $5.2-9.6$ & (7) \\
\hline SDME-GC-FID & $20-100$ & $0.1-50$ & - & 20.0 & $3.5-8$ & (8) \\
\hline HF-LPME-GC-MS & $5-100$ & $0.5-10$ & - & 5.0 & $4-19$ & (9) \\
\hline DLLME-GC-MS & $2-8$ & $0.02-100$ & $681-889$ & 5.0 & $4.6-6.8$ & (11) \\
\hline DLLME-HPLC-VWD & $640-1800$ & $5-5000$ & $44-196$ & 5.0 & $4.3-5.9$ & (12) \\
\hline MSPE-GC-FID & 100 & $0.5-500$ & 350 & 400 & $<8.0$ & Proposed method \\
\hline
\end{tabular}

\section{Disc ussion}

Compared with micrometer-sized particles (commercial adsorbent) used in the SPE, the NPs offer a multitude of benefits that make them a better choice. They have significantly higher ratio of surface area-to-volume and a short diffusion route, resulting in higher extraction capacity, rapid dynamics of extraction and higher extraction efficiencies. Moreover, by using superparamagnetic NPs such as $\mathrm{Fe}_{3} \mathrm{O}_{4}$, a shorter analysis time can be achieved due to the magnetically assisted separation of these particles from the sample solution. These particles are attracted to a magnetic field but retain no magnetic charge after the field removal. This property makes them particularly suitable for sample preparation because, in comparison with non-magnetic adsorbents, no centrifuging or filtration of the sample is needed after extraction. Therefore, in recent years, the use of magnetic NPs for sample extraction and preconcentration is gaining many researchers' interest (16). Most of the published articles in this area are related to use surfactant coated magnetic nanoparticles (termed mixed-hemimicelles) as sorbent. Preparation of these type sorbents is simple; however, due to incompatibility use of surfactants with gas chromatography, it is needed to develop new magnetic sorbents to overcome such problems. Due to generality of octadecyl functionalized silica sorbent and also very extensive application of this sorbent in SPE techniques, preparation of octadecyl functionalized magnetic silica nanoparticles as sorbent can be valuable.

Generally, addition of salt enhances extraction of analytes because the salting-out effect can reduce the solubility of the analytes in water and force more of them onto the adsorbent. Increasing the ionic strength also causes the viscosity of aqueous phase to increase, resulting in a decrease in diffusion coefficients of analytes; such increases lead to a decrease in the extraction efficiency. In SPE methods, it is important that by using the least amounts of sorbent, higher extraction recoveries of the analytes can be obtained even from large volumes of the solution. The aim of the optimization of sample volume is to find maximum sample volume in which constant amount of the analyte can be extracted by a using constant amount of the sorbent with relative recoveries higher than $90 \%$. This leads to getting higher enrichment factors (i.e., higher $\mathrm{V}_{\text {donor }} / \mathrm{V}_{\text {acceptor }}$ ) in analysis of the real samples because a larger volume of the real sample contains higher amounts of analyte. It is important to note that handling of large sample volume $(400 \mathrm{~mL})$ in a short time (typically a few minutes) becomes practically feasible only by means of magnetic sorbents due to the magnetically assisted separation of the sorbent (C18-f-m-Si-NPs) because of their easy collection from sample solution.

Based on the obtained results Table 3 , it can be concluded that the present technique in comparison with with some of the techniques reported in the literature for extraction and determination of plasticizers has a high sensitivity, a broad linear range, and favourable precision. Moreover, the method is fast and simple even for large sample volumnes ( $400 \mathrm{~mL})$ because of using magnetic nanoparticles as adsorbent.

Plastics, especially the polyvinyl chloride (PVC), are most widely used materials in the industry due to their excellent properties (i.e., high chemical and mechanical endurance) and a wide range of applications in our daily life (20). Compounds that are lost or migrate from plastics during the use of a 
plastic product include the components of the final plastic such as the residual monomers and additives, as well as compounds used in the manufacturing process (e.g., to initiate polymerization). Compounds are lost from plastics either because they are not chemically bound to the polymer and are, therefore, free to migrate, or because during the use of the plastic product, the plastic becomes distorted, worn or weathered. This can lead to the exposure and loss of previously bound compounds. Exposure to sunlight (especially UVB) can degrade plastics. Increased levels of UVB as a result of the depletion of stratospheric Ozone could, therefore, impact on plastics. Many of the chemicals in use have not been subject to a risk assessment (21). The addition of plasticizers (mainly phthalates and adipates) makes the plastic flexible and popular for packaging, storing and preserving food, water, and so on (22). The most widely used plasticizers in food contact applications are dioctyl phthalate (DEHP) and dioctyl adipate (DOA) (Vinyl Council of Australia, www.vinyl.org.au). The most probable route of human exposure to DEHP is through food, with an average intake of $0.25 \mathrm{mg} / \mathrm{d}$ (23) and being migrated from plastics during the processing and storage of the main source (24).

Diffusion of chemical substances from polymers is a very complex process, and is dependent on several parameters, such as concentration of substances in packaging film and food, nature of the foods, temperature, and the time period over which duration of contact occurs. During the process of diffusion, these compounds enter into another matrix (such as film or food), which changes their concentration in both the packaging film and food. Although chemical compound migration is mainly a diffusion-controlled process, it is equally important to consider the mechanism of convection and chemical reactions (22).

The obtained results in this research showed that as stated by Bhunia et al. (22), temperature and storage time have a significant effect on the migration of DEHP. At the same study, Salah et al. (25) determined the presence of phthalates in 10 different brands of bottled water available in Saudi markets and stored under different conditions. Dimethyl phthalate (DMP), diethylphthalate (DEP), di-n-butyl phthalate (DBP), benzyl butyl phthalate (BBP) and diethyl hexyl phthalate (DEHP) were measured by headspace solid-phase microextraction followed by gas chromatography- mass spectrometer detector. They found that most of the phthalates were detected in the selected bottled water samples that might be either leached from the plastic packaging materials or contamination during the bottling processes. Bottled waters stored at $4^{\circ} \mathrm{C}$ contained higher levels of DMP, DEP, BBP and DEHP than those stored at room temperature and outdoors. On the other hand, the levels of DMP, DEP and BBP were significantly lower in the bottled waters stored at room temperature than those stored outdoors. They concluded that temperature and sunlight play a role in the degradation of phthalates within time.

Yamini et al. developed two microextraction techniques for the extraction and preconcentration of di-(2-ethylhexyl) phthalate (DEHP) and di-(2ethylhexyl) adipate (DEHA) from the mineral water samples (8). They applied these two methods for the analysis of two plasticizers in different water samples; two target plasticizers were found in the bottled mineral water after the expiring time and the boiling water was exposed to a polyethylene vial. Accordingly, development of sensitive and reliable analytical methods to analyze trace amounts of plasticizers in different water samples is necessary.

\section{Financial disclosure}

The author declared no financial interest.

\section{Funding/Support}

The study did not receive any financial support.

\section{References}

1. Staples CA, Peterson DR, Parkerton TF, Adams WJ. The environmental fate of phthalate esters: a literature review. Chemosphere 1997; 35:667-749.

2. Balafas D, Shaw KJ, Whitfield FB. Phthalate and adipate esters in Australian packaging materials. Food Chem 1999; 65: 279-287.

3. Arcadi FA, Costa C, Imperatore C, Marchese A, Rapisarda A, Salemi M, Trimarchi GR, Costa G. Oral toxicity of bis(2-ethylhexyl) phthalate during pregnancy and suckling in the Long-Evans rat. Food Chem Toxicol 1998; 36: 963-970.

4. Tyler CR, Jobling S, Sumpter JP Endocrine disruption in wildlife: a critical review of the evidence. Crit Rev Toxicol 1998; 28:319-361.

5. National Primary Drinking Water Regulations, Federal register, Part 12, 40 CFR Part 141, US Environmental Protection Agency, Washington, DC, 1 July 1991, p. 395.

6. Lorz PM, Towae FK, Enke W, Jackh R, Bhargava N, Ullmann'sEncyclopedia of Industrial Chemistry, Wiley VCH, Weinheim 7, 2002.

7. Yamini Y, Ghambarian M, Khalili-Zanjani MR, Faraji M, Shariati S Comparison of solidification of floating drop and homogenous liquid-liquid microextractions for the 
extraction of two plasticizers from the water kept in PET bottles. J Sep Sci 2009; 32: 3201-3208.

8. Batlle R, Nerín C. Application of single-drop microextraction to the determination of dialkyl phthalate esters in food simulants. J Chromatogr A 2004; 1045:2935.

9. Psillakis E, Kalogerakis N. Hollow-fibre liquid-phase microextraction of phthalate esters from water. J Chromatogr A 2003; 999: 145-153.

10. Zhang Y, Lee HK. Low-density solvent-based vortexassisted surfactant-enhanced-emulsification liquid-liquid microextraction combined with gas chromatographymass spectrometry for the fast determination of phthalate esters in bottled water. J Chromatogr A 2013; 1274:2835 .

11. Farahani H, Norouzi $\mathrm{P}$, Dinarvand $\mathrm{R}$, Ganjali MR. Development of dispersive liquid-liquid microextraction combined with gas chromatography-mass spectrometry as a simple, rapid and highly sensitive method for the determination of phthalate esters in water samples. J Chromatogr A 2007; 1172:105-112.

12. Liang $\mathrm{P}, \mathrm{Xu} \mathrm{J}, \mathrm{Li}$ Q. Application of dispersive liquidliquid microextraction and high performance liquid chromatography for the determination of three phthalate esters in water samples. Anal Chim Acta 2008; 609:5358.

13. Zhang H, Chen X, Jiang X Determination of phthalate esters in water samples by ionic liquid cold-induced aggregation dispersive liquid-liquid microextraction coupled with high-performance liquid chromatography. Anal Chim Acta 2009; 689: 137-142.

14. Ranjbari E, Hadjmohammadi MR. Magnetic stirringassisted dispersive liquid-liquid microextraction followed by high performance liquid chromatography for determination of phthalate esters in drinking and environmental water samples. Talanta 2012; 100: 447453.

15. Zhao RS, Wang X, Yuan JP, Lin JM. Investigation of feasibility of bamboo charcoal as solid-phase extraction adsorbent for the enrichment and determination of four phthalate esters in environmental water samples. J Chromatogr.A 2008; 1183:15-20.
16. Faraji M, Yamini Y, Rezaee M. Magnetic nanoparticles: Synthesis, stabilization, functionalization, characterization, and applications. J Iran Chem Soc 2010; $7 ; 1-37$.

17. Keyhanian F, Shariati S, Faraji M, Hesabi M. Magnetite nanoparticles with surface modification for removal of methyl violet from aqueous solutions, Arab J Chem 2011, doi:10.1016/j.arabjc.2011.04.012.

18. Faraji M, Yamini Y, Saleh A, Rezaee M, Ghambarian M, Hassani R. A nanoparticle-based solid-phase extraction procedure followed by flow injection inductively coupled plasma-optical emission spectrometry to determine some heavy metal ions in water samples. Anal Chim Acta 2010; 659: 172-177.

19. Yamini Y, Faraji M, Adeli M, Magnetic silica nanomaterials for solid-phase extraction combined with dispersive liquid-liquid microextraction of ultra-trace quantities of plasticizers, Microchimica Acta 2015; 182: 1491-1499.

20. Cano JM, Maran ML, Sanchez A, Hernandis V. Determination of adipate plasticizers in poly(vinyl chloride) by microwave-assisted extraction. J Chromatogr A 2002; 963: 401-409.

21. Sarria-Vidal M, Simal-Gandara J, Nijssen B, Recent Res Dev Agric Food Chem 1998; 2: 781-786.

22. Bhunia K, Sablani SS, Tang J, Rasco B. Migration of chemical compounds from packaging polymers during microwave, conventional heat treatment, and storage. Compr Rev Food Sci Food Saf 2013; 12: 523-545.

23. Koch HM, Drexler H, Angerer J. An estimation of the daily intake of di(2-ethylhexyl)phthalate (DEHP) and other phthalates in the general population. Int $\mathrm{J}$ Hyg Environ Health 2003; 206: 77-83.

24. Lopez-Cervantes J, Paseiro-Losada P. Determination of bisphenol $\mathrm{A}$ in, and its migration from, PVC stretch film used for food packaging. Food Addit. Contam. 2003; 20: 596-606.

25. Al-Saleh I, Shinwari N, Alsabbaheen A. Phthalates residues in plastic bottled waters. J Toxicol Sci 2011; 36: 469-478. 